\title{
Oxytocin: Vom Geburts- zum Sozialhormon
}

\section{Zur hormonellen Regierbarkeit von Soziabilität aka Gesellschaft}

\author{
Xenia Steinbach und Sabine Maasen
}

Oxytocin: From a Hormone for Birth to a Social Hormone. The Hormonal Governance of Sociability aka Society

In the mass media, the hormone Oxytocin is currently being debated as the biochemical basis of sociability and a powerful neuropharmacological solution for (re-)establishing societal cohesion. Given its beginning as a ,bodyhormone' early in the 20th century, this article will trace the extraordinary career of Oxytocin from a regulator of birth to a regulator of society. What makes so strong a claim intelligible and acceptable? Our analysis of the scientific discourse on Oxytocin (1906-1990), the mass media discourse since the 1990s, and its repercussions for the scientific discourse during the same period, suggest a series of re-configurations of scientific theories and practices, as well as of the conception of the substance itself. Oxytocin became established in the first half of the 20th century, and as a neurohormone as early as the 1950s, yet during the following decades attracted little scientific attention. Only following the mass media's focus on the suggested effects of Oxytocin on love and bonding did the substance increasingly become the focus of empirical research. This work argues that the reception of Oxytocin as a potential neurohormonal basis for individual sociability strongly relies on the mass media discourses, biopolitical linkages that had already been made in the first half of the 20th century aiming at the regulation of life, and a technoscientific mode of research on Oxytocin. At their intersection Oxytocin emerged as a social hormone.

Keywords: Oxytocin, Neurohormone, Sociality, Medialization, Biopolitics

In massenmedialen Darstellungen wird das Hormon Oxytocin gegenwärtig als biochemische Basis von Sozialität und wirkmächtiger neuropharmakologischer Lösungsansatz für die (Wieder-)Herstellung der gesellschaftlichen Kohäsion verhandelt. Mit Blick auf die ursprüngliche Bedeutung des Hormons als „Körperhormon“ zu Beginn des 20. Jahrhunderts soll im vorliegenden Artikel die außergewöhnliche Karriere von Oxytocin vom Regulator des Geburtsvorgangs hin zum Regulator der Gesellschaft nachgezeichnet werden. Woraus bezieht eine solch voraussetzungsvolle Behauptung ihre Intelligibilität und Akzeptabilität? Unsere Analyse des wissenschaftlichen Diskurses um Oxytocin (1906-1990), des massenmedialen Diskurses seit den 1990er Jahren sowie dessen Rückwirkungen auf den wissenschaftlichen Diskurs im gleichen Zeitraum verweist auf eine Serie von Re-Konfigurationen von wissenschaftlichen Theorien und Praktiken, sowie der Konzeption der Substanz an sich. Nachdem es sich in der ersten Hälfte des 20. Jahrhunderts etabliert, wird Oxytocin bereits in den 1950er Jahren zum Neurohormon, findet in den folgenden Jahrzehnten jedoch kaum wissenschaftliche Aufmerksamkeit. Erst im Zuge des massenmedialen Interesses für die postulierten Wirkungen des Hormons in Zusammenhang mit Liebe und Bindung gerät die Substanz zunehmend in den Fokus empirischer Forschung. Die Rezeption von Oxytocin als neurohormonelle Basis der individuellen Soziabilität speist sich zum einen aus dem massenmedialen Diskurs, zum anderen aus bereits in der ersten Hälfte des 20. Jahrhunderts gemachten biopolitischen Verknüpfungen, die auf die Regulierung des Lebendigen abzielen, sowie aus einem technowissenschaftlichen Modus der Oxytocinforschung: an ihrem Schnittpunkt avanciert Oxytocin zum Sozialhormon, so unsere These. 


\section{Oxytocin: Kitt der Gesellschaft}

Ignoranten und Ichlinge bestimmen unseren Alltag, das Klima wird rauer. [...] Sozialforscher sehen den Kitt der Gemeinschaft bröckeln. [...] Was ist los mit uns? Die Stiftung für Zukunftsfragen spricht von einer hilflosen Gesellschaft, in der die Menschen immer häufiger neben - statt miteinander lebten. [...] Wie schön, dass es wenigstens eine Arznei gegen Asozialität gibt. Der Botenstoff Oxytocin nämlich baut Stress in unserem Hirn ab und fördert prosoziales Verhalten. Eine Firma in Florida füllte das Mittel vor einigen Jahren in Flaschen und verkauft es seither als „Liquid Trust“: ein Spray, das Menschen wieder menschlicher machen soll. (Schindler 2012a: 28f.)

„Oxytocin ist zu einem kleinen Star in der Verhaltensforschung geworden" stellt Roger Nickl im Unimagazin der Universität Zürich im Jahr 2006 zu Recht fest (Nickl 2006: 36). Spätestens seit Anfang der 2000er Jahre findet man eine stetig wachsende Anzahl internationaler wissenschaftlicher Publikationen zur Bedeutung des Neurohormons Oxytocin in Zusammenhang mit sozialem Verhalten aber auch in vielen anderen Kontexten - eine Publikationsflut, die sich nicht einzig durch die generelle Expansion des wissenschaftlichen Systems erklären lässt. ${ }^{1}$

Als vielversprechendes Therapeutikum wird Oxytocin in klinischen Studien im Hinblick auf seine Wirksamkeit bei Autismus, Borderline-Persönlichkeitsstörungen, Sozialer Phobie und zahlreichen weiteren Erkrankungen untersucht (Andari et al. 2010; Guastella et al. 2009; Heinrichs et al. 2009), und zwar in Psychologie, Psychiatrie, Medizin, Physiologie, Genetik bis hin zur Ökonomie. Insbesondere in seiner synthetischen Form steht Oxytocin, ein kleines Peptidhormon aus neun Aminosäuren, dessen Wirkungen heute unter anderem im Gehirn verortet werden, gegenwärtig im Fokus der medizinischen Forschung: Es verspricht psychische Erkrankungen zu therapieren, die mit Störungen der individuellen Bindungsfähigkeit einhergehen (Meyer-Lindenberg et al. 2011). Zugleich erfährt es seit etwa zwei Jahrzehnten eine enorme massenmediale Aufmerksamkeit: Unter Titeln wie „Sozial-“, „Liebes-“ oder „Kuschelhormon“ wird es in den Massenmedien als biochemisches Korrelat menschlicher Soziabilität diskutiert (Baier 2006; Schäfer 2015: 15).

Als „Arznei gegen Asozialität“ und „Spray, das Menschen wieder menschlicher machen soll“ (Schindler 2012a: 28f.), wird zugleich ein Problem (Desintegration der Gesellschaft) und ein biotechnologischer Lösungsansatz behauptet. Denn Soziabilität oder die Fähigkeit zur Gemeinschaftlichkeit des Individuums gilt als Schlüssel zur Integration der Gesellschaft. Die neuropharmakologische Intervention setzt genau an 
der mangelnden Soziabilität des Individuums an. Gesteigerte Soziabilität therapiert Individuen und integriert die Gesellschaft. Die erstaunliche Intelligibilität und Akzeptabilität dieser Behauptung bedarf, finden wir, einer Erklärung. Wir vermuten zunächst, dass sich diese aus drei unterschiedlichen Trends speist.

Erstens läßt sich beobachten, dass in der Gegenwart nicht länger „die Gesellschaft“, sondern „das Leben“ zur primären Kategorie und zum Angelpunkt individueller und politischer Bearbeitung erhoben wird. Bezeichnend dafür ist die Weise, in der in Folge biowissenschaftlicher Innovationen der lebendige Körper heute weniger als organisches Substrat, denn als molekulare Software begriffen wird, die „gelesen“ und „umgeschrieben“ werden kann. Biopolitisch stellt sich deshalb die Frage nach der Evidenz von hormonell-technologisch modulierbarem Leben innerhalb dieser politisch-technischen Konstellation (Agamben 2002).

Zweitens ereignet sich auch eine Re-Konfiguration der Lebenswissenschaften zu sogenannten Technowissenschaften. In dieser Formation findet zunehmend eine Verquickung von Erforschung, Entwicklung und experimentellem Einsatz zur Analyse und vielfältigen Interventionen in das Lebendige statt (Haraway 1995; Nordmann 2005) und dies in „messy networks of research and development, industry and society" (Weber 2010: 18) - and the mass media, wie wir ergänzen würden. Als Akteur zugleich materieller wie diskursiver Natur (Haraway 2004) kommt dem Hormon Oxytocin dabei die Funktion zu, einerseits auf der Ebene der Hormonwirkung, andererseits auf der Ebene bedeutungsvoller Konnotationen das Narrativ der Selbst- und Fremdregulierungsfähigkeit von Soziabilität auszuformulieren. Es ist Element einer Technowissenschaftskultur.

Drittens können die schlagzeilenträchtigen Titel, zu denen die gegenwärtige Oxytocinforschung Anlass gibt, als das Resultat der von Bourdieu analysierten Politiken des Wissenschaftsfeldes gesehen werden, in deren Kontext Aufmerksamkeit zu den wertvollsten Kapitalvermögen des modernen Wissenschaftsbetriebs gehört (Bourdieu 1975). Daher rekonstruieren wir in diesem Aufsatz die rezente Evidenz dieses Zusammenhangs auch als einen Effekt der "Medialisierung" von Wissenschaft sowie deren Rückwirkung auf Wissenschaft selbst (Weingart 2005).

Am Schnittpunkt von Biopolitik, Technowissenschaftskultur und Medialisierung soll im Folgenden die Karriere von Oxytocin als eine Serie von Re-Konfigurationen nachgezeichnet werden, in denen sich sowohl biologische Regulierungstheorien und -praktiken ändern als auch das, was jeweils als Leben adressiert wird. Während Oxytocin in der ersten Hälfte des 20. Jahrhunderts als hormoneller Regulator des Geburtsvorgangs und „Körperhormon" ${ }^{2}$ etabliert wird, profiliert es sich in den 1950er Jahren als ein Neurohormon, dessen Wirkungen auch im Gehirn verortet werden, 
bis es in den letzten drei Jahrzehnten zum Sozialhormon avanciert und über den wissenschaftlich-medizinischen Diskurs hinaus qua Technologisierung (Nasenspray) selbst- und sozial-regulative Imaginationen anregt. Dabei weitet sich zugleich beständig der Interventionsbereich aus: von der Mutter-Kind-Dyade, auf das Paar, auf die Gesellschaft und von dort wieder zurück auf das Individuum - zunehmend reguliert im Modus des Lebendigen.

Im Sinne Lucy Suchmans stoßen wir auf immer neue Konfigurationsarbeit: „An orientation to configuration reminds us to reanimate the figures that populate our socio-material imaginaries and practices, to examine the relations that they hold in place and the labours that sustain them" (Suchman 2012: 58). Die immer beweglichen Relationierungen werden besonders deutlich, wenn man den Blick auf historisch verschiedene diskursive Konfigurationen des Hormons Oxytocin richtet.

Die folgende Studie ergänzt Forschungen aus der Wissensgeschichte sowie der Medialisierungsforschung. Die Produktion von Wissen über Hormone und das dabei relevante Zusammenwirken von Akteuren ${ }^{3}$, Institutionen, wissenschaftlichen Theorien und Praktiken sowie weiterer zeitspezifischer Faktoren ist bereits Gegenstand wissens(chafts)historischer Arbeiten, denen ein „Verständnis von Wissensproduktion als diskursive Praxis“ gemein ist (Haller 2012: 23). Wir teilen wichtige ihrer Ausgangspunkte und Einsichten.

Mit dem Fokus auf sogenannte "Wirkstoffe“ - eine im deutschsprachigen Raum in der ersten Hälfte des 20. Jahrhunderts gängige Sammelbezeichnung für Vitamine, Enzyme und Hormone - skizziert Heiko Stoff die „Geschichte [...] leistungsstarker, in kleinsten Mengen wirksamer, in Bezug auf die Behebung von Mangelzuständen, Mangelsituationen und Mangelkrankheiten etablierter chemischer Agentien zur biologischen Regulierung leistungsfähiger Körper." (Stoff 2012: 11) Er hebt die kokonstitutive Beziehung von Wirkstoffen und Gesellschaft hervor: „Die Geschichte der Wirkstoffe ist auch ein Exempel dafür, wie soziale Probleme hergestellt und in technowissenschaftliche Lösungsmöglichkeiten übersetzt werden“ (Stoff 2012: 8).

Mit Heiko Stoff und auch Lea Haller teilen wir zudem die Absage an eine lineare Geschichtsschreibung sowie an eine szientistische Vorstellung von „der Entwicklung einer pharmazeutischen Substanz im Sinne des rational drug design" (Haller 2012: 16). Vielmehr verstehen auch wir die Etablierung und den Einsatz der Hormone und anderer bioaktiver Substanzen im Sinne Foucaults als „Produkte eines zeitspezifischen Dispositivs“ (Haller 2012: 17; Stoff 2012: 21). Wie Stoff, Haller, Nelly Oudshoorn und Christina Ratmoko gehen wir daher von der konstitutiven Bedeutung des politischen und wirtschaftlichen Kontextes für wissenschaftliches Wissen über die Hormone 
aus - auch wenn er in der vorliegenden Analyse nachrangig behandelt wird (Oudshoorn 2000; Ratmoko 2010). Uns geht es primär um die allmähliche Verfertigung eines Sozialhomons über seine (techno)wissenschaftliche Konfigurierung und dynamisiert durch dessen Medialisierung.

Entsprechend orientieren wir uns an der Medialisierungsforschung. Medialisierung beruht auf der Annahme, dass Wissenschaft einerseits zum "Gegenstand medialer Dauerbeobachtung" avanciert ist, und dass sich die Wissenschaft andererseits zunehmend an massenmedialen Relevanzkriterien und öffentlichen Erwartungen orientiert (Weingart 2005: 28). Als Ursachen für solche Dynamiken werden zum einen die wachsende Bedeutung der massenmedialen Kommunikation in der Gesellschaft genannt, die gegenwärtig auch Wissenschaft und Technologie erfasst: Sie haben news value erlangt. Zum anderen wird auf den wachsenden Legitimationsdruck der Wissenschaft gegenüber Politik, Wirtschaft und der Gesellschaft, sowie Fördergebern verwiesen. ${ }^{4}$

Seit einiger Zeit geraten auch die Lebenswissenschaften in den Fokus der Medialisierungsforschung (Schäfer 2007; Rödder 2009). Insbesondere für den Bereich der Neurowissenschaften wird festgestellt, dass sie sich in forcierter und instrumenteller Weise in den Medien präsentieren und sich immer selbstverständlicher an medialen Erwartungen orientieren (Allgaier et al. 2012; Heinemann 2012; Peters et al. 2013).

Informiert durch Wissensgeschichte und Medialisierungsforschung beobachten wir eine Serie von Re-Konfigurationen des Oxytocin vom Körperzum Neurohormon bzw. vom Geburts- zum Sozialhormon. Die Analyse des wissenschaftlichen Diskurses bis 1990 zeigt, dass das Hormon Oxytocin im medizinischen Diskurs bereits über ein Jahrhundert lang bekannt ist und dass viele für innovativ gehaltene Aspekte ebenfalls schon vor vielen Jahrzehnten, wenn auch in anderer diskursiver Formation, entwickelt wurden. Es ist jedoch erst die Medialisierung der Oxytocinforschung, die den Zusammenhang der synthetisch regulierbaren Liebe zum Kind, zur Partnerin, zur Gruppe, zur Gesellschaft hervorbringt. Die These lautet, dass diskursive Verknüpfungen, die bereits in der ersten Hälfte des 20. Jahrhunderts gemacht wurden, sich erst um die Jahrtausendwende im Medium der Medialisierung zum Phänomen "Sozialhormon Oxytocin“ konfigurieren. Die Rückwirkung der Medialisierung auf die Oxytocinforschung ist, dass sie sich - nach wie vor auf schmaler Befundlage - zu weitreichenden Behauptungen veranlasst sieht: Aus dem Geburtshormon wird schließlich auch in der Forschungspublikation umstandslos ein Liebes- und Sozialhormon. ${ }^{5}$

Während die genannten wissenshistorischen Arbeiten sich in etwa auf den Zeitraum 1900-1970 beschränken, setzen die entscheidenden Re-Konfigurationen des Oxytocin zum Sozialhormon in der letzten Dekade des 
20. Jahrhunderts an - genau zum Zeitpunkt der verstärkten Medialisierung dieser Forschung. Um die vorangehenden Transformationen sowie die dann einsetzenden Re-Konfigurationsbewegungen zu profilieren, gehen wir zunächst dem wissenschaftlichen Diskurs um Hormone im Zeitraum zwischen 1906 und 1950 nach. Hier zeigen wir, wie Oxytocin im Rahmen der sich etablierenden neuen Theorie der „Inneren Sekretion“ zunächst als Geburtshormon konzipiert wird. Im zweiten Abschnitt gehen wir den diskursiven Verknüpfungen nach, die zwischen hormonellen Wirkungen und psychischen Vorgängen bereits in den 1920er Jahren gemacht werden. Die dritte Momentaufnahme des historischen Rückblicks richtet sich auf die in den 1950er Jahren aufkommende Theorie der „Neurosekretion“: Sie leitet die Re-Konfiguration von Oxytocin vom Körper- zum neurowissenschaftlich beforschbaren Gehirnhormon ein. Daran anschließend wenden wir uns der Medialisierung des Oxytocin seit dem Jahr $1990 \mathrm{zu}$ - hier vollziehen sich die entscheidenden diskursiven Transformationen des Oxytocin zum Sozialhormon, um sodann den Blick auf die Rückwirkungen der Medialisierung auf die Oxytocinforschung zu richten. Der Epilog reflektiert kurz auf den Zusammenhang von news value und bio value der Oxytocinforschung.

\section{Oxytocin wird zum Geburtshormon (1906-1950)}

„Noch ganz am Anfang“ stehe die Erforschung des Hormons Oxytocin, heißt es in einem Interview mit einem renommierten Wissenschaftler auf diesem Gebiet aus dem Jahr 2014 (Stein 2014). Ob in massenmedialen Darstellungen oder wissenschaftlichen Publikationen - allseits wird die Neuheit und Brisanz der Erkenntnisse um die Substanz bekräftigt (Wolf 2013; Faber 2014). Dabei ist das Hormon Oxytocin keineswegs eine wissenschaftliche Neuentdeckung, sondern ein bereits seit den Geburtsstunden der Endokrinologie bekanntes Körperhormon und hormonelles Therapeutikum.

Im Jahr 1906 wird Oxytocin ${ }^{6}$ erstmals als eine in der Hypophyse (Hirnanhangdrüse) gebildete biochemische Substanz beschrieben (Dale 1906), welche die Geburtswehen einleiten und für den Milcheinschuss nach der Geburt verantwortlich sein soll (Dale 1909). Damit ist Oxytocin zu Beginn des 20. Jahrhunderts eine von vielen neuartigen Substanzen, deren Wirkungen man in Experimenten zwar beobachten und lokalisieren kann. Allerdings hat man zu dieser Zeit weder eine Vorstellung von ihrer chemischen Struktur, noch ist man in der Lage, diese Substanzen in Reinform zu gewinnen. Dennoch verweisen ihre Wirkungen auf ein neues physiologi- 
sches Modell, welches das zu dieser Zeit etablierte Körperkonzept grundlegend in Frage stellt.

Bevor man die Vorstellung von einer biochemischen Regulation entwickelt, dominiert ein „mechanistisch-reduktionistisches Körpermodell“ den physiologischen Diskurs des 19. Jahrhunderts (Sarasin \& Tanner 1998: 27). Im Mittelpunkt physiologischer Theorien stehen Leistungen der Nerven, die die wesentlichen Körperfunktionen nach einem Reiz-ReaktionsSchema steuern (Haller et al. 2014: 364). Im auslaufenden 19. Jahrhundert wird der menschliche Körper zunehmend problematisiert: Industrialisierung und Verstädterung provoziere körperliche Überreizung und Schwächung der Gesellschaftsmitglieder (Radkau 1998). Dies stellt Politik und Medizin vor die Herausforderung, wissenschaftliche Lösungen für weitreichende gesellschaftliche Problemlagen zu finden (Stoff 2003: 224).

Die Koproduktion von Politik, Medizin und Gesellschaft wird jedoch erst effektiv als sich in den letzten Dekaden des 19. Jahrhunderts zunehmend das Experimentieren sowie die Manipulation und Modulation von Organismen als gängige Praxis medizin-wissenschaftlicher Erkenntnisgewinnung durchsetzt: Die medizinische Forschung konfiguriert sich als „aktive Wissenschaft" und intervenierendes Programm (Stoff 2012: 12; KnorrCetina 1984). Die nun systematisch durchgeführten Experimente an Tieren, die Entnahme von Organen und Anwendung ihrer Extrakte auf andere Lebewesen oder Gewebe und insbesondere die experimentelle Erzeugung von Mangelzuständen und Missbildungen weisen auf die Existenz hochwirksamer biochemischer Substanzen hin, deren Effekte mit physiologischen Modellen dieser Zeit nicht zu erklären sind (Böttcher 1963).

Erst die seinerzeit berühmten wie umstrittenen Selbstversuche des Physiologen Charles Édouard Brown-Séquard Ende der 1880er Jahre verschaffen in medizinischen Fachkreisen Aufmerksamkeit für die These der chemischen Regulation körperlicher Vorgänge (Rechter 1997). Seine Theorie der „Inneren Sekretion“ basiert auf der Vorstellung von Drüsen, die spezifische Substanzen über den Blutkreislauf an unterschiedliche Organe des Körpers transportieren um dort ihre Wirkung zu entfalten. Während die innerwissenschaftliche Diskussion um die korrekte Bezeichnung der zunächst unsichtbar verbleibenden biochemischen Agentien bis in die 1930er Jahre anhält, setzt sich im öffentlichen Diskurs schnell der von Ernest Starling im Jahr 1905 vorgeschlagene Begriff „Hormone“ durch und wird schließlich auch in der Wissenschaft zur gängigen Bezeichnung (Starling 1905). ${ }^{7}$

Ende des 19. Jahrhunderts rückt so die Gestaltbarkeit des Lebendigen in den Fokus der Wissenschaft (Pauly 1987) und regt sogleich Phantasien und Visionen des Machbaren an - auch über den wissenschaftlichen Kontext hinaus (Nordlund 2007; Rechter 1997). ${ }^{8}$ 
Dabei ist der Diskurs um Hormone von Beginn an stets an politische Fragen und gesellschaftliche Problemlagen geknüpft. Das neue Konzept der biochemischen Regulierung „verweist [...] ebenso auf kybernetische Steuerungsprozesse wie auf Techniken der Normierung der Bevölkerung und des Körpers. Regulierung ist danach eine das Lebendige politisierende Form der Regierung, der Steuerung sowohl körperlicher Leistungen als auch der Kontrolle der Bevölkerung insgesamt" (Stoff 2012: 9). Damit rückt „das Leben“, nun verstanden als biochemisch regulierte und regulierbare Einheit, in den Fokus von (bio)politischen Regierungsstrategien, an denen auch Hormone beteiligt werden. Zahlreiche Krankheiten werden als Folgen hormoneller Mangelerscheinungen oder der Überproduktion verstanden und für hormonell therapierbar erklärt. Dieses Deutungsschema birgt ein nahezu grenzenloses Reservoir an neuartigen Interventionsmöglichkeiten - der drohenden Gesellschaftskrise stehen vielversprechende wissenschaftliche Lösungsansätze entgegen, die eine bessere und gesündere Zukunft in Aussicht stellen (Nordlund 2007; Rechter 1997).

Im Kontext dieser Entwicklungen etabliert sich Oxytocin als wirkmächtiges Körperhormon. Der Geburtsvorgang wird nun zu einem hormonell gesteuerten und steuerbaren Ereignis, was sogleich auch materielle Effekte hat: Bereits lange vor der Isolierung und der synthetischen Herstellung der Substanz durch Vincent du Vigneaud im Jahr 1953 werden die postulierten Wirkungen von Oxytocin im Rahmen der Geburtsmedizin genutzt. Durch die Gabe von tierischen Hypophysenextrakten oder durch die Injektion von aus gebärenden Frauen gewonnenem Blut werden zu schwache Wehen verstärkt oder die Geburt künstlich eingeleitet. Als in den 1960er Jahren schließlich ein industriell hergestelltes hormonelles Präparat mit Oxytocin auf den Markt kommt, ist Oxytocin bereits längst als Geburtshormon bekannt. Trotz seiner Lokalisierung in der Hypophyse werden dem Hormon Oxytocin in der ersten Hälfte des 20. Jahrhunderts allerdings keine psychischen Wirkungen zugeschrieben - obgleich dies bereits in den 1920er Jahren Gegenstand von Forschung und Popularisierung ist.

\section{Hormone und Psyche (1920-1950)}

Der wissenschaftliche Diskurs Anfang des 20. Jahrhunderts ist von Grabenkämpfen verschiedener Disziplinen und Erklärungsansätze des Psychischen bestimmt. Während sich die Freud'sche Psychoanalyse und der Behaviorismus mit umfassenden Deutungsangeboten jedoch aufwändiger Therapiemethodik großer Beliebtheit erfreuen, stellt die neue hormonelle Physiologie einfache Erklärungen und angeblich unkomplizierte Interven- 
tionsmöglichkeiten in Aussicht (Rechter 1997: 5). Auch hier wirft die Idee des Oxytocin als Sozialhormon - und zwar als Vision - seine Schatten voraus.

Ein besonders anschauliches Beispiel für die Verbreitung hormoneller Theorien im Zusammenhang mit Persönlichkeit und Verhalten im deutschsprachigem Raum stellen die Schriften des Arztes und populären Wissenschaftsautors Dr. med. et phil. Gerhard Venzmer zwischen 1920 und 1950 dar. In den Kosmos-Heftchen der Frankch'schen Verlagshandlung erweitert Venzmer die zeitgenössisch populäre Konstitutionslehre Ernst Kretschmers (Kretschmer 1921) um eine biochemische Deutungsebene und entwickelt eine hormonell fundierte Typenlehre (Venzmer 1933: 22f.) In dieser Konfiguration stellt die individuelle hormonelle Konstitution die biologische Grundlage der menschlichen Persönlichkeit dar - eine Erweiterung mit weitreichenden Folgen: Während die Konstitutionslehre vormals lediglich als Erklärungsansatz diente, erlaubt die hormonelle Deutungsebene neuartige Möglichkeiten der Manipulation. Bemerkenswert ist, dass sich in Venzmers Darstellungen neben Abhandlungen über die Schilddrüse, die in der ersten Hälfte des 20. Jahrhunderts als „Drüse der Gemütsbewegungen" gilt (Venzmer 1933: 180), eine explizite Verknüpfung zwischen einem nicht näher bezeichneten in der Hypophyse gebildeten „Vorderlappenhormon" und menschlichen Verhaltensweisen und Wesenszügen findet:

Die Heilkunde weiß bereits die auf das Psychische gerichtete Wirkung des Vorderlappenhormons zu benutzen; wo z. B. der „hypophysäre Charakter" übermäßige, krankhafte Formen annimmt, läßt die Zufuhr von Vorderlappen-Hormon die Kranken oft unverkennbar lebhafter, energischer und tatenfroher werden; niedergedrücktes, müdes, unlustiges und schläfriges Wesen bessert sich. [... A Auch Angstzustände, feindselige und gesperrte Haltung gegen die Nebenmenschen, gesellschaftsfeindliche Einstellung sowie charakterliche Härte, Starrheit und Unbeugsamkeit lassen sich - wenn hormonell bedingt - bisweilen durch geeignete Hypophysenbehandlung erstaunlich bessern, indem die Einreihungsfreudigkeit und Zugänglichkeit zunehmen. (Venzmer 1933: 187)

Individuelle soziale Passung und gesellschaftliche Integration sind, so die Überzeugung, durch pharmakologische Mittel herbeizuführen. Gleichwohl: Noch gibt es die Vorstellung eines Sozialhormons nicht. Die Hormone der Hirnanhangdrüse üben ihren physiologischen Einfluss nur indirekt auf psychische Vorgänge aus. Wie eine „übergeordnete Schaltzentrale“ agiert die Hypophyse als „Anlasser der Geschlechtshormone“ (Venzmer 1933: 77), indem sie die Aktivität der in den Geschlechtsorganen liegenden Keimdrüsen steuert. Dass die Tätigkeit der Hypophyse ihrerseits durch 
Hormone und andere chemische Substanzen des Hypothalamus reguliert wird, und dass Hormone ihren Einfluss auf Emotionen und Verhalten direkt im Gehirn ausüben können, ist zu dieser Zeit nicht bekannt. Verhaltensweisen und Persönlichkeitseigenschaften werden vielmehr auf die Wirkungen der sogenannten „Mann- und Weibhormone“ zurückgeführt und sind stark geschlechterdichotom codiert (Oudshoorn 2000: 89).

Damit ist eine sozioepistemische Konfiguration angesprochen, die den frühen Diskurs um Hormone von Beginn an prägt und von anderen Autorinnen bereits ausführlich behandelt wird: ${ }^{9}$ Aufgekommen in einer Zeit der zunehmend instabil werdenden Geschlechterrollen (Planert 2007: 194), wird die frühe Hormonforschung $\mathrm{zu}$ einem politischen Instrument der Wiederherstellung einer erwünschten gesellschaftlichen Ordnung. So werden zunächst ambivalente Ergebnisse aus der Erforschung der Sexualhormone in ein Deutungsraster integriert, das die biochemische Dichotomie der Geschlechter untermauern soll (Ratmoko 2010: 13; Oudshoorn 2000: 89) und noch entscheidender: die Möglichkeit in Aussicht stellt, diese sich nun als natürlich darstellende Ordnung mittels hormoneller Präparate herzustellen (Haraway 1995; Sengoopta 2006).

Obgleich viele der Versprechen der frühen Endokrinologie nie eingelöst werden - mit der Theorie der Inneren Sekretion wird das Modell der biochemischen Regulation psychischer Vorgänge bereits in der ersten Hälfte des 20. Jahrhunderts plausibel. Zudem erfährt die Endokrinologie von Beginn an eine enorme wissenschaftliche wie öffentliche Aufmerksamkeit: Es sind die weitreichenden Interventionsmöglichkeiten die das neue Konzept der biochemischen Regulierung gesellschaftspolitisch interessant machen und der Endokrinologie eine Vormachtstellung gegenüber anderen Ansätzen verschaffen. So wird das vormals dominante Nervensystem zwar teilweise in das neue physiologische Modell integriert - Nerven dienen im neuen Körperkonzept als elektrisches System der Reizwahrnehmung und Weiterleitung und somit als Bindeglied zwischen dem hormonellen System und der Außenwelt. Da den beiden Systemen jedoch eine grundlegend unterschiedliche Funktionsweise zugeschrieben wird, betrachtet man das hormonelle und das nervliche System als weitgehend voneinander getrennt. Entsprechend wird auch die Hypophyse, obwohl sie im Gehirn lokalisiert ist, als Bestandteil des hormonellen Systems verstanden. Dem Gehirn wird zwar weiterhin das Attribut eingeräumt, "Sitz der Seele“ zu sein (Hagner 2008), doch rückt das Gehirn in dieser Zeit gewissermaßen in den Hintergrund und das hormonelle System dominiert sowohl in wissenschaftlichen als auch populären Darstellungen das körperliche und geistige Geschehen. In der ersten Hälfte des 20. Jahrhunderts bildet die Endokrinologie die zentrale Projektionsfläche für koproduktive Visionen einens biochemisch regulierten und regulierbaren Menschen und damit 
einer Gesellschaft, deren drohender Degeneration und Schwächung nun entgegengewirkt werden kann.

\section{Oxytocin wird zum Neurohormon (1950-1990)}

Die Theorie der „Neurosekretion“ transformiert in den 1950er Jahren die Vorstellung von der Funktionsweise des hormonellen sowie des nervlichen Systems grundlegend. Danach werden Hormone nicht nur durch spezielle, über den Organismus verteilte Drüsen produziert, sondern können auch durch Nervenzellen im Gehirn ausgeschüttet werden. Sie werden nicht nur an den Blutkreislauf abgegeben, sondern übertragen Informationen auch im Gehirn und entfalten ihre Wirkung innerhalb des zentralen Nervensystems (Scharrer \& Scharrer 1954). Diese Theorie steht in den 1930er Jahren, als sie erstmals von Ernst und Berta Scharrer postuliert wird, im Widerspruch zur der konzeptionellen Trennung zwischen dem Nervensystem und dem hormonellen System und wird in Fachkreisen deshalb zunächst nicht anerkannt (Scharrer 1937). Den optischen Beweis für die Theorie der Neurosekretion erbringt fast zwanzig Jahre später die in den 1940er Jahren entwickelte Technologie der histologischen Färbung. Sie unterstützt die Beobachtung, dass Oxytocin nicht, wie zunächst angenommen, durch die Hirnanhangdrüse, sondern durch Nervenzellen im Hypothalamus produziert wird. Von dort aus kann Oxytocin über Nervenbahnen zur Hypophyse transportiert, gespeichert und anschließend an den Blutkreislauf abgegeben werden oder zentral im Gehirn wirken. Damit avanciert Oxytocin zu einem der ersten bekannten „Neurohormone“ (Fehm 2001; Korf 2014).

Mit dieser Verortung der Wirkungen von Oxytocin auch im Gehirn erfährt es eine Erweiterung: es ist nun nicht mehr nur ein Körperhormon, sondern auch ein Gehirnhormon. Für das Gehirn als ein die geistigen Vorgänge steuerndes Organ (Hagner 2008) spielen Hormone bis in die 1950er Jahre keine Rolle. Erst mit der Theorie der Neurosekretion werden hormonelle Substanzen allmählich zum Gegenstand der Hirnforschung.

Kurz: Mit der Vorstellung einer biochemischen Regulierung körperlicher Vorgänge durch die Theorie der Inneren Sekretion wird Oxytocin zu Beginn des 20. Jahrhunderts zu einem Körperhormon und etabliert sich anschließend als Therapeutikum im Rahmen der Geburtsmedizin. Mit der Anerkennung der Theorie der Neurosekretion in den 1950er Jahren erweitert sich Oxytocin zum Gehirnhormon und wird damit zu einem potenziellen Regulator auch geistiger Vorgänge. Die neurowissenschaftliche Verhaltensforschung greift Oxytocin jedoch erst in den 1980/90er Jahren 
auf, wonach es rasant an wissenschaftlichem und öffentlichem Interesse gewinnt. Wodurch erklärt sich diese stark verzögert einsetzende Aufmerksamkeit für Oxytocin in seiner neuen Rolle als Neurohormon?

Zunächst zeichnet sich Mitte des 20. Jahrhunderts eine Verschiebung der Deutungshierarchie der Disziplinen im Hinblick auf die Funktionsweise des Körpers und der Psyche ab. Während sich aus der Hirnforschung die moderne Neurowissenschaft herausbildet, deren neue Methoden und Technologien wachsende Beachtung finden, wird die in der ersten Hälfte des 20. Jahrhunderts nahezu euphorisch betriebene Hormonforschung zunehmend prekär (Stoff 2012: 282f.). Nicht eingelöste Versprechen, enttäuschte Erwartungen und insbesondere erschreckende Nebenwirkungen - so beispielsweise die krebserregenden Wirkungen von Östrogen-Präparaten - werfen seit den 1950er Jahren einen dunklen Schatten auf die vermeintlichen „Wunderstoffe“ (Stoff 2012: 298). Ein Blick auf die massenmediale Berichterstattung zeigt, dass auch Oxytocin in den 1970er Jahren im Rahmen der Debatte um die "Programmierte Geburt" - eine umstrittene Praxis der künstlichen Geburtseinleitung, die sich nach den pragmatischen Erfordernissen der Krankenhäuser richtet - unter Beschuss gerät (Schreiber 1980). Nicht nur gelten Hormone zunehmend als potenziell gefährlich: In der Nachkriegszeit verlieren biologistische Erklärungen angesichts der rassenideologischen Verbrechen generell an Popularität und erleben erst in den letzten Dekaden des 20. Jahrhunderts im Zuge der aufsteigenden Lebenswissenschaften einen erneuten Aufschwung in Wissenschaft und Medien (Linke 2007: 12).

Die Vermutung liegt nahe, dass Oxytocin im Kontext dieser Ereignisse nicht sogleich zu einem Hormon der Liebe und Bindung, ja zum Sozialhormon avancieren kann, da diskursive Gelegenheitsstrukturen für eine solche Transformation fehlen. Für diese Vermutung spricht auch die Beobachtung, dass der aktuelle Diskurs um Oxytocin als Sozialhormon den kritischen Diskurs um Oxytocin als Medikament zur künstlichen Einleitung der Geburt nahezu gänzlich ausblendet.

Aus Sicht der Naturwissenschaften erklärt sich das verspätet einsetzende Interesse dagegen aus einem rein forschungspragmatischen Umstand: Lange Zeit entziehen sich die postulierten Wirkmechanismen aufgrund der sogenannten Blut-Hirn-Schranke einer extensiven experimentellen Erforschung (Fehm 2001: 210). ${ }^{10}$ Intravenös injiziertes Oxytocin kann die BlutHirn-Schranke nicht passieren. Lediglich die Injektion der Substanz direkt in das Gehirn erlaubt die Umgehung der Blut-Hirn-Schranke - eine Praxis, die in Bezug auf Menschen jedoch nicht erlaubt ist. Deshalb stellt die Arbeit an Tiermodellen zunächst die einzige Möglichkeit dar, Oxytocin auf seine Wirksamkeit als Neurohormon hin zu beforschen. 
Betrachtet man jedoch die Anfang der 1990er Jahre rasant ansteigende öffentliche Aufmerksamkeit für die Substanz, wird deutlich, dass sie sich vor allem an der behaupteten Wirksamkeit des Hormons auf das menschliche Sozialverhalten entzündet. Dies geschieht, nota bene, obwohl sich humanexperimentelle Techniken mit Oxytocin und der Einsatz einer intranasalen Applikationstechnik zur Überwindung der Blut-Hirn-Schranke erst nach der Jahrtausendwende allmählich etablieren und selbst bis heute nur wenige dieser Behauptungen wissenschaftlich belegt sind (Leng \& Ludwig 2015; Quintana \& Wooley 2015). Es ist der massenmediale Diskurs, der schon zuvor Oxytocin als neurohormonelles Therapeutikum für menschliche Sozialität verhandelt und damit eine erneute Re-Konfiguration von sozioepristemischen und materiellen Komponenten des OxytocinDiskurses einläutet.

Ein schlaglichtartiger Überblick der populären Berichterstattung über Oxytocin seit Beginn der 1990er Jahre zeigt wie Sozialität der Nahbeziehung zwischen Mutter und Kind, der intimen Beziehungen zwischen Erwachsenen, auf Ebene der Interaktionen in Gruppen, sowie in neuer Weise bezogen auf das Individuum selbst, als Effekt des Neurohormons Oxytocin gedeutet wird. Die Neurohormonalisierung menschlicher Sozialität erfolgt dabei im Modus der sich stetig überbietenden Wirksamkeitsbehauptungen der Substanz. Übersetzt in einzelne, wissenschaftlich messbare Komponenten wie Vertrauen, Empathie und Bindung, und begründet in der neurohormonellen Konstitution des Individuums, wird Sozialität dabei zunehmend als hormonell regulierbar gedacht.

\section{Oxytocin wird zum Sozialhormon (1990-2017)}

Oxytocin ist ein Elixier, das bei bemerkenswert vielen Vorgängen in Kopf und Körper eine Rolle spielt: Oxytocin = Vertrauen, Liebe, Treue, Zärtlichkeit, Bindung, Einfühlungsvermögen, Verständnis, Großzügigkeit - die Liste der Erklärungsmuster ist lang. (Anonym 2010a)

Schon ein erster Blick auf die massenmediale Berichterstattung um Oxytocin der letzten drei Jahrzehnte zeigt, dass die vielfältigen Wirkungen des Hormons stets um die individuelle Soziabilität arrangiert sind. Das massenmediale Narrativ lautet: Als Effekt des körpereigenen Oxytocin-Haushalts vollziehe sich die Entwicklung der individuellen Soziabilität bereits im frühkindlichen Alter durch die bindungsstiftende Wirkung von Oxytocin bei der Mutter-Kind-Dyade. Oxytocin sei aber auch ausschlaggebend für das Eingehen von Paarbindungen und die Entstehung von Liebe 
im Erwachsenenalter sowie für die generelle Fähigkeit des Individuums, sich in Gruppen und in der Gesellschaft zu integrieren (Anonym 2012; Wolff 2008). Ein Mangel an körpereigener Oxytocin-Produktion soll zu Defiziten der sozialen Fertigkeiten und damit nicht nur zu geringerer Lebensqualität für die Betroffenen, sondern auch zu einer Gefährdung für den gesamtgesellschaftlichen Zusammenhalt führen (Förster 2012). Insbesondere in Darstellungen seit den 2000er Jahren zeichnet sich dabei zunehmend das neoliberale Imperativ der (hormonellen) Selbstoptimierung (Schaper-Rinkel 2012) im Dienste gesellschaftlicher Integration ab. Synthetische Präparate oder körpereigenes Oxytocin generierende Selbstpraktiken versprechen demnach individuelles Glück und eine bessere Gesellschaft. Dieser Fokus auf neuropharmakologisch begründete Handlungsund Eingriffsoptionen forciert nicht nur verstärkte technowissenschaftliche Forschung (Nordmann 2005), sondern auch die Ausbildung einer Technowissenschaftskultur, in der auch im Alltag neurohormonell induzierte Soziabilitätssteigerungen wünschbar sind und praktiziert werden. Wie wird die diskursive Verknüpfung von neuropharmakologischen Technologien mit Oxytocin und menschlicher Sozialität nun plausibilisiert?

Die Vermutung ist, dass die Konstruktion von Oxytocin als Sozialhormon durch Mechanismen der Verstärkung in der massenmedialen Berichterstattung angetrieben wird, die nun ein entscheidendes Element in der Konfiguration werden. Verstärkung ist „eine alte Kommunikationstechnik für unbeweisbare oder schwer beweisbare Behauptungen" (Luhmann 2007: 218). Auch die Evidenz der vielfältigen Bindungswirkungen von Oxytocin wird durch stetige Wiederholungen, Übertreibungen und wechselseitige Bezugnahmen forciert: durch „zirkuläre Zirkulation der Nachricht“ (Bourdieu 1998: 74). Rhetorische Verallgemeinerungen stellen ein weiteres wichtiges Moment der Konfigurationsarbeit dar, was sich bereits in den ältesten Artikeln über Oxytocin im Kontext von Sozialität abzeichnet.

Obwohl sich die ersten Berichte auf wenige Studien an Mäusen beziehen, die eine kopulationsanregende Wirkung von synthetischem Oxytocin bei den Tieren belegen sollen, werden bereits hier Bezüge hergestellt, die weit über diese wissenschaftlichen Erkenntnisse hinausgehen. So liest man beispielsweise in einem Artikel aus dem Jahr 1991:

Das chemisch unscheinbare Oxytocin, meint die amerikanische Zoologie-Professorin Sue Carter, sei „im Hinblick auf die Evolution ein altes, uraltes Hormon“; es habe, indem es Hautkontakte förderte, schon in grauer Vorzeit „die Entwicklung von Sozialbeziehungen“ in Gang gesetzt. Für den Anatomen Gustav F. Jirikowski aus La Jolla in Kalifornien ist Oxytocin eine erste sichtbare „Brücke zwischen Physiologie und Verhalten" - gleichsam ein Band zwischen Körper und Seele, aber auch zwischen den Lebewesen. (Anonym 1991: 240) 
Aussagen wie diese unterstützen die Vorstellung eines biochemischen Mechanismus, der psychische Vorgänge steuern und für die Entstehung von sozialen Beziehungen auch zwischen Menschen, ihren Körpern und Seelen, verantwortlich sein soll. In der neuen Konfiguration erfolgt die hormonelle Steuerung des Psychischen jedoch direkt und nicht mehr auf dem Umweg über die Sexualhormone, wie es in den Narrativen der 1920er Jahre ist. Die evolutionshistorische Referenz suggeriert eine unabweisbare Relevanz der Thematik, deren Plausibilität durch das Auftreten von Experten verstärkt wird - kontrastiv verstärkt durch die Unscheinbarkeit des Oxytocin. Die Formulierung „erste sichtbare Brücke“ ignoriert indessen vorangehende Diskurse - eine Strategie, die die Berichterstattung um Oxytocin und auch die Neurowissenschaften im Allgemeinen durchzieht. ${ }^{11}$

Einer mechanistischen Logik folgend, wird das Neurohormon zunehmend zur biochemischen Basis der Mutterliebe erklärt und ein monokausaler Wirkungszusammenhang behauptet:

Erste Hinweise darauf, wie diese starke, frühe Bindung zwischen Mutter und Kind entsteht, fanden zwei amerikanische Wissenschaftler im Jahr 1968, als sie jungen, kinderlosen Ratten Blut von Artgenossinnen injizierten, die gerade Nachwuchs bekommen hatten. Die Tiere begannen sofort, Nester zu bauen, sie fütterten und putzten fremde Rattenbabys. Elf Jahre später fand man heraus, dass ein einziges Hormon, Oxytocin, dieses Verhalten auslöst. [...] Beim Menschen ist das nicht anders als bei Ziegen [...]. Ihr Körper ist also in den Stunden nach der Geburt vom Liebeshormon Oxytocin überschwemmt. (Baier 2006)

In dieser Darstellung fungiert Oxytocin als wirkmächtiges „Liebeshormon“, das auf natürliche Weise im Körper Gebärender ausgeschüttet wird und zur Bindung an das Neugeborene führt. Ganz selbstverständlich werden dabei erneut Erkenntnisse aus Expermenten an Nagetieren auf das menschliche Bindungsverhalten übertragen. ${ }^{12}$ Das Narrativ des Liebesmoleküls schließt dabei an die psychologische Bindungstheorie nach John Bowlby an (Bowlby 1969): Ein liebevoller Umgang der Mutter mit ihrem Neugeborenen gilt als entscheidender Faktor für eine gesunde Entwicklung der kindlichen Psyche und der individuellen Soziabilität. Diese Theorie wird durch das Neurohormon Oxytocin um eine biochemische Deutungsebene erweitert und dient gleichzeitig als argumentativer Ausgangspunkt für weitere Überbietungsdiskurse.

Denn in Folge etabliert sich Oxytocin als das Liebes- und Bindungshormon nicht nur für die Beziehung zwischen Mutter und Kind, sondern auch für die Beziehung zwischen erwachsenen Partnern. Vergleichbar der Geburt, soll die Produktion des Hormons auch durch Geschlechtsverkehr 
und zärtliche Berührungen angeregt werden: „Beim Orgasmus, so haben die Experten ermittelt, rauscht eine wahre Oxytocin-Flutwelle durch die Blutkanäle; sie sorgt, nach dem Absturz vom Lustgipfel, für eine entspannte und friedliche Stimmung, die soziale Bindungen fester knüpft" heißt es im Spiegel (Anonym 1991: 240).

Oxytocin „knüpft“ Bindungen jedoch nicht nur „fester“, vielmehr avanciert es in den 1990er Jahren im populärwissenschaftlichen Diskurs um Liebe und Paarbindung zum "Treuehormon“ - bei einer bestimmten Wühlmausart soll das Hormon für die Entstehung lebenslanger Paarbindungen verantwortlich sein (Anonym 1993: 228f.). Die Beobachtungen aus den Experimenten werden in den massenmedialen Berichten jedoch in ein Narrativ eingebettet, das deutlich auf menschliche Paarbeziehungen referiert. Mit Begriffen wie „stabile Familienverhältnisse“ und „Liebesleben“ werden monogame Fortpflanzungsgemeinschaften von Mäusen mit der lebenslangen Ehe von Menschen gleichgesetzt (Ebd.). Solche Formulierungen verwischen sukzessiv die Grenzen zwischen wissenschaftlich fundierten Erkenntnissen und massenmedialen Behauptungen (Shen 2015). Ganz im Sinne der „zirkulären Zirkulation der Nachricht“ (Bourdieu 1998: 74) verstärkt die stete Wiederholung zudem ihre Plausibilität. Damit avanciert Oxytocin im öffentlichen Diskurs bereits in den 1990er Jahren zum Liebesund Treuehormon, obwohl zu dieser Zeit noch keinerlei Untersuchungen zu den Wirkungen der Substanz bei Menschen vorliegen ${ }^{13}$ und die Substanz im wissenschaftlichen Diskurs zunächst weiterhin wenig Beachtung findet.

Dies ändert sich jedoch in der ersten Dekade des neuen Jahrtausends. Mit den ersten Experimenten an Menschen treten neue neuropharmakologische Technologien in den Vordergrund: Synthetisches Oxytocin wird nun intranasal in Form von Nasensprays verabreicht, da man davon ausgeht, dass es in dieser Form die Blut-Hirn-Schranke passieren und im zentralen Nervensystem wirken kann (Born et al. 2002). An diesem Punkt erweitert sich das Repertoire der Wirksamkeitsbehauptungen um die sozialitätstiftende Wirkung des Oxytocin deutlich, da die Substanz nun zunehmend in den Fokus medizinischer Forschung gerät: Bindungsstörungen, Empathiemangel und soziale Ängste sind seine Anwendungsbereiche (Anonym 2010b; von Bredow 2005). Die Berichterstattung über diese Forschung fällt dabei beachtlich positiv aus. Die Angst vor Nebenwirkungen und Manipulation wird stets ausgeräumt:

„Es macht Menschen ja nicht willenlos." Szenarien wie das eines Parlaments, dessen Abgeordnete durch Gase aus der Klimaanlage gefügig gemacht werden, verweist er [Oxytocin-Forscher Markus Heinrichs] ins Reich der Fantasie. „Wir müssen eine sehr hohe Dosis direkt in 
die Nase sprühen, damit das funktioniert." Das gehe nur deshalb ohne Risiko, weil Oxytocin ja eine körpereigene Substanz ist: „Das Zeug hat kaum Nebenwirkungen." (Stöcker 2005)

Der Verweis auf die Unschädlichkeit des Oxytocin und das Argument seines körpereigenen Ursprungs verkennt indessen die Ambivalenz des Einsatzes von Neurotechnologien zur (Wieder-)Herstellung von Soziabilität. Es wird unterschlagen, dass synthetisches Oxytocin die natürliche Substanz lediglich nachahmt. Dies unterscheidet Oxytocin von anderen neuromanipulativen Substanzen, die im Rahmen der Debatte um Neuro-Enhancement seit einigen Jahren höchst kontrovers diskutiert werden. Während kognitionssteigernde Mittel wie Ritalin oder stimmungsaufhellende Mittel wie Prozac als potentiell gefährlicher Eingriff in das natürliche Funktionieren des Körpers diskutiert werden (Ach \& Lüttenberg 2011: 234), wird Oxytocin als Substanz körpereigenen Ursprungs positioniert. Diese Verkennung des Artifiziellen stellt einen wichtigen Mechanismus der Plausibilisierung von neuropharmakologischen Technologien, Oxytocin und Sozialität dar. Der vorangegange Diskurs der 1970er und 1980er Jahre um Oxytocin als Medikament zur künstlichen, technizistischen und gefährlichen Geburtseinleitung wird dabei gänzlich ausgeblendet.

Auch der potentielle Einsatz von Oxytocin-Präparaten zur Erhaltung von Partnerschaften löst weder Bedenken hinsichtlich der Wirksamkeit des Hormons noch seiner Verabreichung als Nasenspray aus. Vielmehr geht es um ethische Fragen nach der Qualität der pharmakologisch induzierten "Neuroliebe“. Hier finden sich zwar Bedenken gegenüber der "Authentizität" der durch Oxytocin-Präparate manipulierten Liebesempfindung (Bethge et al. 2014: 116). Dennoch sprechen sich sowohl Neurowissenschaftlerinnen als auch Bioethikern in der Öffentlichkeit meist für den Einsatz von neuropharmakologischen Technologien aus: „Liebe, die von Arzneien befeuert wird, ist nicht anders als Liebe, die von Martinis, schlauem Wortgeplänkel oder gutem Sex befördert wird“, sagt Larry Young. „Das Gefühl ist dasselbe.“ (Bethge et al. 2014: 116)

Solche medialen Statements von Wissenschaftlern verweisen auf die koproduktive Rolle dieser Akteure. Die funktionale Gleichstellung von synthetischem mit körpereigenem Oxytocin verschafft den daran anknüpfenden medialen Behauptungen weitere Plausibilität und Akzeptabilität. Die in diesem Kontext wiederkehrende Dramatisierung von stetig wachsenden Scheidungsraten verstärkt zudem die Dringlichkeit von Interventionen zur (Wieder-)Herstellung der gesellschaftlichen Kohäsion - neuerdings auch abseits von neuropharmakologischen Technologien: „Es gibt genügend natürliche Wege, die Oxytocin-Freisetzung zu steigern', ergänzt Hurlemann. 
,Umarmungen oder andere zärtliche Berührungen zum Beispiel““ (Stein 2014).

Insbesondere am Beispiel der „Kuschelpartys“ (Steinecke 2008: 33) wird deutlich, wie sich Selbsttechnologie und Hormon nochmals anders positionieren. Dem durch zunehmende Technisierung und moderne Kommunikationstechnologien bedingten Mangel an „echter" zwischenmenschlicher Nähe und der daraus resultierenden Fragmentierung der Gesellschaft soll nun durch Selbsttechnologien, die körpereigenes, sozialitätstiftendes Oxytocin produzieren, entgegengewirkt werden. Erneut zeigt sich deutlich, an welchen Problemzusammenhang der Diskurs um Oxytocin rückgebunden wird: Oxytocin-Technologien - ob als materielle oder als Selbsttechnologien - werden kurzerhand als Lösungsansatz für gesamtgesellschaftliche Probleme verhandelt.

Daraus begründet sich vermutlich auch eine Besonderheit im medialen Diskurs um Oxytocin: Bis auf wenige Ausnahmen fällt die Berichterstattung gänzlich positiv aus. Selbst wissenschaftliche Publikationen, in denen erstmals negative Wirkungen des Neurohormons behauptet werden, erfahren im Gros der Massenmedien eine unerwartet positive Resonanz: „Oxytocin verstärke Ethnozentrismus, also die Feindseligkeit gegenüber Personen, die nicht zur eigenen Gruppe gehören, während der Kontakt innerhalb der Gruppe besser werde“ berichtet der Focus im Jahr 2012 (Tenzer 2012), worauf weitere Berichte über Studien folgen, die für eine Aggressivität steigernde Wirkung von Oxytocin sprechen sollen (Kara 2016). Was in manchen Beiträgen zunächst als „Die dunkle Seite des Kuschelhormons“ (Anonym 2014; Stein 2014) rezipiert wird und der etablierten Imagination als Sozialhormon entgegen zu stehen scheint, wirkt bei genauer Betrachtung tatsächlich als weitere verstärkende Komponente des Diskurses. Denn obwohl negativ konnotiert, beruht sie im Kern auf der Behauptung, Oxytocin habe eine regulative Wirkung für das Sozialverhalten in Gruppen auch wenn im Modus eines in-group/out-group Schemas nicht notwendigerweise harmonisierend. Damit erreichen die Wirksamkeitsbehauptungen eine neue Ebene - die der Beziehungen in Gruppen. Oxytocin plausibilisiert sich dadurch weiter als Sozialhormon.

Doch so gegensätzlich die Versuchsergebnisse erscheinen - Kooperation hier, Aggression dort -, sie widersprechen einander nicht. Im Grunde verhielten sich die Probanden immer gleich: nämlich so, dass es der eigenen Gruppe nutzt - notfalls auf Kosten anderer. [...] Oxytocin ist also ein Kuschelhormon - aber auch ein Kampfstoff. Das Bild der Star-Substanz wird differenzierter. (Kara 2016: 29)

Zusammenfassend lässt sich festhalten, dass die diskursive Spannung im Fall des Oxytocin nicht aus der Umstrittenheit der Substanz resultiert, und 
somit weder aus einer wissenschaftlichen noch öffentlichen Kontroverse, sondern aus der Kopplung an zahllose gesellschaftsrelevante Probleme. Von weit verbreiteten psychischen Erkrankungen, über Bindungsunfähigkeit und Vereinsamung, bis hin zur Gefährdung der gesamtgesellschaftlichen Integration - in der Konfiguration von Oxytocin als Sozialhormon zeichnen die Medien das Bild einer harmlosen „natürlichen“ Substanz, die Stabilität in eine instabil gewordene Gesellschaft $\mathrm{zu}$ bringen verspricht und ungeachtet ihrer Technizität den Status des Natürlichen und Guten erhält (Schindler 2012b). Diese Dynamik kann jedoch nicht allein als Produkt massenmedialer Aktivitäten verstanden werden, zeichnet sich hier doch bereits ab, dass auch wissenschaftlichen Akteur_innen keineswegs eine passive Rolle bei der Konstruktion des Oxytocin als Sozialhormon zukommt. Der nächste Abschnitt beleuchtet die Rückwirkungen der Medialisierung auf den wissenschaftlichen Diskurs und verdeutlicht, dass die Medialisierung der Oxytocinforschung die Forschung selbst keineswegs unberührt lässt.

\section{Rückwirkungen der Medialisierung auf die Oxytocinforschung (1990-2017)}

In der letzten Dekade des 20. Jahrhunderts und insbesondere seit der Jahrtausendwende zeichnen sich zunehmend Veränderungen im wissenschaftlichen Diskurs um Oxytocin ab, die im Folgenden im Kontext der verstärkten Medialisierung dieses Forschungsfeldes diskutiert werden sollen. Zum einen häufen sich wissenschaftliche Publikationen aus der Oxytocinforschung, deren Rhetorik sich zunehmend medialen Inszenierungslogiken annähert (Imhof 2006) sowie Publikationen, die ungewöhnlich weitreichende thematische Anschlüsse an gesellschaftlich relevante Themenkomplexe aufweisen. Zum anderen beeinflussen massenmediale Selektionskriterien erkennbar die Wahl der Forschungsthemen. Des weiteren kann man eine steigende Präsenz von Wissenschaftlernnen in den Massenmedien beobachten. Schließlich zeichnen sich Rückwirkungen der Medialisierung auch in der Weiterentwicklung von Interventionstechnologien ab, die im massenmedialen Diskurs bereits angekündigt und erst anschließend zum Gegenstand von Forschung werden.

An zwei Beispielen sollen im Folgenden Effekte der Medialisierung auf der Ebene wissenschaftlicher Veröffentlichungen illustriert werden. Die Publikationen der amerikanischen Verhaltensforschergruppe um Dr. Sue Carter zur Rolle von Oxytocin für das Sozialverhalten von Wühlmäusen zu Beginn der 1990er Jahre liefert ein anschauliches Beispiel. Ihre Tier- 
experimente deuten Carter und Kolleginnen als Belege für eine das Bindungsverhalten modulierende Wirkung des Neurohormons: Oxytocin soll an der Ausbildung monogamer Fortpflanzungsgemeinschaften bei spezifischen Wühlmäusen beteiligt sein (Carter et al. 1992). In dieser Arbeit finden sich keinerlei Verweise auf die Gültigkeit der Ergebnisse für das Phänomen menschlicher Sozialität. In der massenmedialen Berichterstattung zu diesen Studien (siehe oben) häufen sich dagegen Begriffe wie „MäuseEhe“, „Liebesleben“, „stabile Familienverhältnisse“ und „Pärchen“ (Anonym 1993: 228f.) - Begriffe, durch die sich ein semantisches Feld der explizit menschlichen Beziehungen eröffnet. Im Jahr 1998 publiziert Carter daraufhin einen Review-Artikel zum damaligen Forschungsstand der Wirksamkeit von Neurohormonen für soziales Verhalten bei Tieren mit dem Titel „Neuroendocrine perspectives on social attachment and love“ (Carter 1998). Darin spricht sie erstmals von „Liebe“ durch Oxytocingabe. Zwar räumt sie ein, dass es kaum Belege für Wirkungen des Hormons auf das menschliche Verhalten gebe und das komplexe hypothetische Konzept der Liebe sich schwerlich auf konkrete, experimentell erforschbare Verhaltensweisen reduzieren ließe. Dennoch betrachtet sie das Fortpflanzungs- sowie Nachwuchspflegeverhalten als Effekte neurohormoneller Regulation durch Oxytocin und definiert sie als essentielle Komponenten des Phänomens der Liebe. Ihr Schluss: „Therefore, the analysis of parental and sexual behaviors, which can be studied in a variety of species, also offers insight into the neurophysiology of love" (Carter 1998: 780) liefert die wissenschaftliche Basis für Behauptungen, die im massenmedialen Diskurs bereits zuvor gemacht wurden. Ihre Formulierung birgt zudem implizit die Aussicht auf Regulierbarkeit von sozialer Bindungsfähigkeit - ein Aspekt, der eine enorme gesellschaftliche Relevanz der Oxytocinforschung suggeriert.

Die Orientierung an massenmedialen Selektions- und Darstellungslogiken ist im Jahr 2005 bereits explizite Strategie wissenschaftlicher Fachzeitschriften. So liefert die renommierte britische Fachzeitschrift Nature ein zweites Beispiel für Effekte der Medialisierung von Wissenschaft. Sie unterhält die Rubrik Letters, in der kurze und explizit an Wissenschaftler anderer Fachbereiche adressierte Beiträge veröffentlicht werden. Autoren von Letters werden dazu angehalten, die generelle Relevanz der eigenen Forschung zu erläutern und die Inhalte auch für fachfremde Wissenschaftlerinnen verständlich zu vermitteln. Martina Franzen zeigt, dass solche Strategien multidisziplinärer Fachzeitschriften einen Fokus auf massenmediale Erfolgskriterien zulasten wissenschaftlicher Rigorosität begünstigen können (Franzen 2011). Die vorliegende Untersuchung richtet sich demgegenüber auf den konstruktiven Aspekt solcher Medialisierungsphänomene: Inwiefern tragen solche Artikel zur Plausibilisierung des Zusammenhangs 
von neurohormonellen Technologien, Oxytocin und menschlicher Sozialität bei?

Der Artikel „Oxytocin increases trust in humans“ beginnt mit den Worten:

Trust pervades human societies. Trust is indispensable in friendship, love, families and organizations, and plays a key role in economic exchange and politics. [...] Much recent evidence indicates that trust contributes to economic, political and social success. Little is known, however, about the biological basis of trust among humans. Here we show that intranasal administration of oxytocin, [...] causes a substantial increase in trust among humans, thereby greatly increasing the benefits from social interactions. (Kosfeld et al. 2005: 673)

Zwischenmenschliches Vertrauen soll nicht nur unentbehrlich für Nahbeziehungen sein, sondern auch für das Funktionieren von Wirtschaftsbeziehungen, der Politik, bis hin zur Funktionsweise der gesamten Gesellschaft. Die neurobiologischen Grundlagen des Vertrauens erlauben es indessen es pharmakologisch - durch intranasal verabreichtes Oxytocin zu steigern. An solchen Formulierungen wird erneut deutlich, welch weitreichende gesellschaftspolitische Dimension der Oxytocinforschung und den von ihr in Aussicht gestellten Interventionstechnologien nun auch in wissenschaftskommunikativen Publikationen für ein multidisziplinäres $\mathrm{Pu}$ blikum beigemessen wird.

Im abschließenden Abschnitt des Artikels findet sich sodann eine Argumentationsfigur, die sich als Integration von medialen und wissenschaftlichen Relevanzkriterien lesen läßt:

However, our findings may also have positive clinical implications for patients with mental disorders that are associated with social dysfunctions (for example, social phobia or autism). [...] Thus, our results might lead to fertile research on the role of oxytocin in several mental health disorders with major public health significance. (Kosfeld et al. 2005: 675)

Der Ausblick lenkt den Fokus auf den klinischen Anwendungskontext den Bereich, der den experimentellen Einsatz der Substanz bei Menschen begründet. Dieses doppelte Versprechen aus klinischem Nutzen im Kontext psychiatrischer Erkrankungen einerseits und einer über den medizinischen Kontext hinausreichenden Wirksamkeit andererseits, trägt wesentlich zur Legitimation der Oxytocinforschung bei: Förderungswürdigkeit und news value folgen.

Die hier skizzierten Beispiele lassen auf einen sich selbst verstärkenden Prozess schließen: Die massenmediale Berichterstattung zieht ein gestei- 
gertes öffentliches Interesse an der Oxytocinforschung nach sich (Agenda Setting) und befördert damit im Gegenzug die Forschungsaktivitäten, die in der Öffentlichkeit als relevant gewertet werden - eine typische Rückwirkung der Medialisierung der Oxytocinforschung auf die Oxytocinforschung selbst.

Weitere Beobachtungen im wissenschaftlichen Oxytocindiskurs unterstützen diese Annahme: Einerseits zeichnet sich ab, dass die öffentliche bzw. mediale Aufmerksamkeit gelegentlich auch Auswirkungen auf die Forschungsthemenwahl und das Experimentdesign hat. Dafür sprechen sich im letzten Jahrzehnt häufende Studien, deren wissenschaftliche Relevanz in Frage gestellt werden kann: Untersuchungen zur Wirkung von Oxytocin auf das Streitverhalten von Paaren (Ditzen et al. 2009), auf das Empfinden von Neid und Schadenfreude (De Dreu et al. 2011; Shalvi \& De Dreu 2014) oder Studien zur Wirksamkeit des Neurohormons auf die politische Gesinnung von Probanden (Merolla et al. 2013). Darin werden Problemzusammenhänge aufgegriffen, die den wissenschaftlichen Kontext weit übersteigen. ${ }^{14}$

Andererseits gibt es in der Oxytocinforschung, wie in anderen Forschungsbereichen auch, immer mehr Vertreter, die über ihre wissenschaftliche Reputation hinaus auch Medienprominenz erlangen - ein Phänomen, das unter dem Begriff „visible scientists“ (Goodell 1977) diskutiert wird (Rödder 2011). ${ }^{15}$ Nicht selten verfassen Wissenschaftler zudem populärwissenschaftliche Bücher, die, wie beispielsweise aus folgender Rezension des Buches der renommierten Oxytocinforscherin Uvnäs Moberg hervorgeht, an die Überbietungsdiskurse der Massenmedien anschließen und diese zusätzlich verstärken: „Es gibt kaum etwas, was Oxytocin nicht kann - den Eindruck bekommt man zumindest, wenn man das Buch der schwedischen Medizinerin Kerstin Uvnäs Moberg liest" (Paschek 2016: 1).

Nicht zuletzt spielt die stete Weiterentwicklung der Interventionsformen eine wichtige Rolle: Während die Erforschung der Wirkungen der synthetischen Substanz im Fokus des wissenschaftlichen Diskurses steht, weisen Wissenschaftlerinnen in medialen Beiträgen vermehrt auf die Möglichkeit hin, eine körpereigene Produktion des Hormons durch bestimmte soziale und Selbsttechnologien erzielen zu können. Dies wird gegenwärtig beispielsweise im Forschungszweig der Neuro-Ökonomie aufgegriffen. Nicht die Grundlagenforschung stellt das Programm der Neuro-Ökonomie dar, vielmehr sind es weitreichende gesellschaftliche Problemlagen, für die konkrete Lösungen gefunden werden sollen, „damit letztendlich Probleme der globalen Wirtschaft besser angegangen werden können“ (Anonym 2017). Die Neuro-Ökonomie inszeniert sich als eine moderne Technowissenschaft im Dienste des Gemeinwohls und rekurriert auf aktuelle Trends und Ereignisse (etwa: Vertrauensverlust in Politik und Wirtschaft, Weltwirtschafts- 
und Flüchtlingskrise). Der Lösungsansatz der von ihr propagierten „Sozialen Neurowissenschaft" lautet: Training der eigenen Empathiefähigkeit durch spezielle Meditationstechniken - diese sollen die körpereigene Sozialhormon-Produktion ankurbeln, die individuelle Soziabilität steigern und damit aus dem für veraltet erklärten „Homo Oconomicus“ einen altrusitischen Marktteilnehmer und ein soziales Gesellschaftsmitglied machen (Heuser 2013; McCall et al. 2014). Die biopolitische Dimension eines mutmaßlich unscheinbaren Hormons gewinnt mit Bezug auf das Wirtschaftsverhalten eine weitere Facette ebenso wie einen neuen Forschungskontext.

\section{Epilog}

Altern, Menopause, Kinderwunsch, Wachstum, aber auch Schüchternheit ${ }^{16}$ oder Bindungsfähigkeit werden seit Beginn der 1990er Jahre verstärkt als biologische Prozesse begriffen, die durch (synthetische) Hormongaben therapiert oder auch einem Enhancement zugeführt werden können. Seit einigen Jahren häufen sich Berichte über den zukünftigen Einsatz von Oxytocinpräparaten für das „Neuro-Enhancement der Liebe“ - eine Thematik, die auch die Aufmerksamkeit der Wissenschaftsethik erhält (Bittner 2011: 53; Savulescu \& Sandberg 2008). Die Erhöhung von Bindungsfähigkeit stellt sich aber auch in den Dienst großer Fragen im Feld von Politik und Wirtschaft.

Was heute als Hormonalisierung von Liebe und Gesellschaft auftritt, gelingt durch den Rekurs auf die Gestaltbarkeit des Lebens durch technowissenschaftliche Regulierung. Sie materialisiert sich im synthetisch herstellbaren und - so die Vision - als effizientes Nasenspray verabreichbaren Oxytocins. Die gegenwärtige Intelligibilität und Akzeptabilität dieses Versprechens auf hormonelle Regierbarkeit von Mutter-Kind-Beziehungen, Paaren, Gruppen, ja der Gesellschaft ergibt sich am Kreuzungspunkt verschiedener epistemischer und technischer Evidenzbehauptungen, die sich auf wissenshistorisch ausgelegte diskursive Verknüpfungen beziehen und durch Medialisierungseffekte ko-produziert sowie dynamisiert werden. Darüber hinaus findet sich dieses Versprechen in der Nachbarschaft bio- und neurotechnologischer Praktiken, die sich ebenfalls gegenwärtig „auf die individuelle Steigerung der Leistungsfähigkeit und sozialen Kompatibilität sowie der volkswirtschaftlichen Optimierung" (Schaper-Rinkel 2012: 86) ausrichten und zunehmend als Selbstpraktiken in die Verantwortung des Einzelnen verlagert werden. Die biopolitischen Anrufungen auch lebenswissenschaftlich informierter Interventionsformen sind bereits eingeführt: Dem Individuum wird, in neosozialer Manier (Lessenich 2003), 
Verantwortung für die Bindungsfähigkeiten in der Gesellschaft zugeschrieben. Hormonelle Interventionen fügen sich hier ein - auch jenseits evidenzgestützter Innovationen als Imaginationen der Selbst- und Fremdregulierung.

Dieses Regime untersteht verschiedenen Selbstüberbietungsdynamiken: jener, die in die Forschung und in die Medialisierung von Forschung eingeschrieben ist, aber auch jener, die durch embodied risk (Kavanagh \& Broom 1998) entsteht. Ein Körper, der einer Hormon(ersatz)behandlung unterzogen wird, gerät in Dynamiken von Prävention und Monitoring. Immer neue Regulierungsrisiken stimulieren neue Forschungs- und Therapiehoffnungen sowie Erwartungen an Modulation und Enhancement. All dies kondensiert zur gegenwärtigen Evidenz hormonell ko-regulierbaren Lebens: „This extensive consumption of hormones takes place at a time in which taking multiple drugs ,for life' is a widely accepted practice“ (Roberts 2007: 111) - mit hohem symbolischen und ökonomischen „biovalue“ (Waldby 2000) einerseits und hohem news value andererseits. Die Lösung mangelnder gesellschaftlicher Kohäsion lautet: Erhöhung der Sozialität. Der Lösungsweg: Erhöhung individueller Soziabilität - unter anderem mithilfe synthetischen oder körpereigenen Oxytocins. Oxytocinforschung: Willkommen am Schnittpunkt von Medialisierung, Biopolitik und Technowissenschaftskultur!

\section{Anmerkungen}

1 Die Datenbanksuche (webofknowledge.com) nach der Anzahl von Publikationen zu den Stichworten „Oxytocin social“ ergibt im Jahr 1990 noch keine Treffer. Im Jahr 1995 findet man 66 Publikationen, im Jahr 2000 sind es 162 Publikationen, im Jahr 2005, sind es 341 Publikationen, fünf Jahre später 820 und im Juni 2017 findet man bereits 2.848 Veröffentlichungen.

2 Der Begriff „Körperhormon“ ist kein biologischer Fachterminus, sondern wird hier zur Abgrenzung zum Begriff „Neurohormon“ bzw. „Gehirnhormon“verwendet und bezieht sich auf einen funktionalen Unterschied: Während Neurohormone ihre Wirkungen im Gehirn entfalten, werden Körperhormone über die Blutbahn zu anderen Organen des Körpers transportiert und wirken dort. In der ersten Hälfte des 20. Jahrhunderts waren Hormone ausschließlich als an verschiedenen Orten des Körpers, jedoch nicht im zentralen Nervensystem wirkende biochemische Substanzen bekannt.

3 Aus Gründen der besseren Lesbarkeit verwenden wir im vorliegenden Text sowohl das generalisierte Femininum als auch das generalisierte Maskulinum.

4 Für Analysen der Art und Häufigkeit von Darstellungen wissenschaftlicher Ereignisse in den Massenmedien siehe Bauer (2011) und Schäfer (2007). Beispielhaft für Untersuchungen zu strukturellen Veränderungen von Forschungsinstitutionen und deren Anpassungstendenzen an massenmediale Anforderungen stehen Peters et al. (2008); Weingart (2011) und Blöbaum et al. (2013). 
5 Der Analysekorpus setzt sich aus wissenschaftlichen Publikationen, massenmedialen Beiträgen und populärwissenschaftlicher Literatur über Hormone im Allgemeinen und Oxytocin im Speziellen zusammen und wurde einer theorem- und thesengeleiteten Diskursanalyse unterzogen.

6 Die Bezeichnung Oxytocin bedeutet in Anlehnung an das Altgriechische „schnelle Geburt".

7 Siehe dazu Berblinger (1928: 7).

8 Zu Visionen der Verjüngung durch Hormongabe siehe Stoff (2004).

9 Exemplarisch sind etwa die Arbeiten von Fausto-Sterling (1985); Haraway (1989); Fox Keller (1984); Oudshoorn (1990); Oudshoorn (2000); Wiesner (2002); Sengoopta (2006); Roberts (2007) und Ratmoko (2010).

10 Als Blut-Hirn-Schranke bezeichnet man eine selektive physiologische Barriere, die den Übertritt von Substanzen zwischen Blutstrom und Gehirn kontrolliert (Behrends et al. 2017).

11 Torsten Heinemann spricht auch von der "Geschichtsvergessenheit" der Neurowissenschaften und deutet dies als entscheidenden Aspekt der Konstruktion des bahnbrechenden Charakters des Forschungsfeldes in der Öffentlichkeit (Heinemann 2012: 105).

12 Kritische Betrachtungen der Übertragung von Forschungsergebnissen von Tieren auf Menschen finden sich bei Kenney und Müller (2016), sowie Nelson (2013) und explizit zu den Monogamie-Studien mit Oxytocin an Mäusen bei Willey und Giordano (2011).

13 Allerdings: Aus Geheimakten der DDR-Dopingforschung, die im Jahr 1991 der Öffentlichkeit zugänglich gemacht wurden, geht hervor, dass Oxytocinpräparate in unterschiedlichen Darreichungsformen bereits seit den 1960er Jahren an Menschen getestet wurden. Diese Forschungsergebnisse finden jedoch bis heute keinen Anklang im wissenschaftlichen Diskurs (Berendonk 1991: 231).

14 Entsprechend problematisch gestaltet sich in solchen Fällen das Experimentaldesign, in dem komplexe soziale Situationen auf wenige kontrollierbare Faktoren reduziert werden. Im medialen Diskurs gewinnen solche Studien häufig an Glaubwürdigkeit und Plausibilität, indem die jeweiligen Wissenschaftler als Experten zu Wort kommen und den Behauptungen aus ihrer Sprecherposition als kompetente Akteure Nachdruck verleihen (Rutherford 2004).

15 So beispielsweise der renommierte deutsche Oxytocinforscher Professor Dr. Markus Heinrichs, der nicht nur über zahlreiche vielzitierte Publikationen in einflussreichen wissenschaftlichen Journals verfügt, sondern auch regelmäßig in Funk- und Fernsehbeiträgen auftritt und als Experte bei nahezu jedem deutschsprachigen Beitrag über Oxytocin zu Wort kommt. Auch die amerikanische Anthropologin Dr. Helen Fisher steht beispielhaft für eine ausgeprägte mediale Präsenz in Zusammenhang mit Oxytocin. Im Unterschied zu Heinrichs ist Fisher jedoch keine zentrale Figur der Oxytocinforschung, sondern bewegt sich wissenschaftlich lediglich in Grenzbereichen zu dieser Forschung, was in medialen Berichten jedoch nicht deutlich wird und die Anerkennung ihrer Sprecherposition als Expertin demnach auch keineswegs einschränkt.

16 Zur Medikalisierung von Schüchternheit und der möglichen Behandlung mit Oxytocinpräparaten siehe Wehling (2016).

\section{Literatur}

Ach, Johann S. und Beate Lüttenberg 2011. Ungleich besser? Zwölf Thesen zur Diskussion über Neuro-Enhancement. In: Willy Viehöver und Peter Wehling (Hg.). Entgrenzung der Medizin. Von der Heilkunst zur Verbesserung des Menschen. Bielefeld: Transcript: 231-250. 
Agamben, Giorgio 2002. Homo sacer - Souveräne Macht und bloßes Leben. Frankfurt a. M.: Suhrkamp.

Allgaier, Joachim, Sharon Dunwoody, Hans Peter Peters, Dominique Brossard und Yin-Yueh Lo 2012. Neurowissenschaften in den Medien: Die Sicht neurowissenschaftlicher Experten. Neuroforum (4): 304-308.

Andari, Elissar, Jean-René Duhamel, Tiziana Zalla, Evelyn Herbrecht, Marion Leboyer und Angela Sirigu 2010. Promoting Social Behavior with Oxytocin in High-Functioning Autism Spectrum Disorders. Proceedings of the National Academy of Sciences of the United States of America (107): 4389-4394.

Anonym 1991. Hormone: Steinzeit im Kopf. Der Spiegel (8): 240.

Anonym 1993. Tiere: Versklavte Nager. Der Spiegel (24): 228-229.

Anonym 2010a. Verhaltensänderung: Hormon Oxytocin macht Männer einfühlsamer. Spiegel Online. URL: http://www.spiegel.de/gesundheit/sex/verhaltensaenderung-hormonoxytocin-macht-maenner-einfuehlsamer-a-844059.html. Zugegriffen: 29.04.2017.

Anonym 2010b. Kuschelhormon: Oxytocin verstärkt Emotionen für Mama. Spiegel Online. URL: http://www.spiegel.de/wissenschaft/mensch/kuschelhormon-oxytocinverstaerkt-emotionen-fuer-mama-a-731822.html. Zugegriffen: 10.11.2017.

Anonym 2012. Anti-Angst-Hormon Oxytocin wird gezielt an seine Wirkorte im Gehirn transportiert. Homepage der Max Planck Gesellschaft. URL: http://www.mpg.de/ 5018735/oxytocin_gehirn. Zugegriffen: 08.02.2016.

Anonym 2014. Das Hormon fürs Soziale. Oxytocin: Therapie gegen Autismus und Schizophrenie? Focus Online. URL: http://www.focus.de/wissen/mensch/psychologie/ dashormon-fuer-s-soziale-oxytocin_id_3997823.html. Zugegriffen: 19.07.2017.

Anonym 2017. Gemeinsam meditieren gegen die Einsamkeit: Neue Form der Meditation verbindet Menschen. Homepage der Max-Planck Gesellschaft. URL: https://www.mpg. de/10895526/kontemplative-dyade. Zugegriffen: 05.01.2017.

Baier, Tina 2006. Mutterliebe: Das stärkste Gefühl entschlüsselt. Spiegel Online. URL: http:// www.spiegel.de/wissenschaft/mensch/mutterliebe-das-staerkste-gefuehl-entschlues selt-a-415306.html. Zugegriffen: 14.05.2006.

Bauer, Martin W. 2011. Public Attention to Science 1820-2010-A “Longue Durée" Picture. In: Simone Rödder, Martina Franzen und Peter Weingart (Hg.). The Sciences Media Connection: Public Communication and its Repercussions. Dordrecht: Springer: 35-57.

Behrends, Jan C., Josef Bischofberger, Rainer Deutzmann, Heimo Ehmke, Stephan Frings, Stephan Grissmer, Markus Hoth, Armin Kurtz, Jens Leipziger, Frank Müller, Claudia Pedain, Jens Rettig, Charlotte Wagner und Erhard Wischmeyer 2017. Physiologie. Stuttgart: Thieme.

Berblinger, Walther 1928. Die Innere Sekretion im Lichte der morphologischen Forschung. Jena: Fischer.

Berendonk, Brigitte 1991. Doping Dokumente: Von der Forschung zum Betrug. Berlin: Springer.

Bethge, Philip, Rafalela von Bredow und Laura Höflinger 2014. Immer jauchzend, nie betrübt. Der Spiegel (33): 110-118.

Bittner, Uta 2011. Neuro-Enhancement der Liebe: Wird die Liebe zu einem medizinisch kontrollierbaren Phänomen? Zeitschrift für medizinische Ethik (57): 53-61.

Blöbaum, Bernd, Andreas M. Scheu, Annika Summ und Anna-Maria Volpers 2013. Medien, Fächer und Politik: Wie Medien forschungspolitische Entscheidungen in verschiedenen wissenschaftlichen Disziplinen beeinflussen. In: Edgar Grande, Dorothea Jansen, Otfried Jarren, Arie Rip, Uwe Schimank und Peter Weingart (Hg.). Neue Governance der Wissenschaft. Reorganisation - externe Anforderungen - Medialisierung. Bielefeld: Transcript: 289-309.

Born, Jan, Tania Lange, Werner Kern, Gerard P. McGregor, Ulrich Bickel und Horst L. Fehm 2002. Sniffing Neuropeptides: A Transnasal Approach to the Human Brain. Nature Neuroscience (5): 514-516.

Böttcher, Helmuth M. 1963. Das Hormonbuch: Die Geschichte der Hormonforschung. Köln: Kiepenheuer \& Witsch.

Bourdieu, Pierre 1975. The Specificity of the Scientific Field and the Social Conditions of the Progress of Reason. Social Science Information (14): 19-47.

Bourdieu, Pierre 1998. Über das Fernsehen. Frankfurt a. M.: Suhrkamp. 
Bowlby, John 1969. Attachment and Loss. Vol. 1: Attachment. New York: Basic Books.

Bredow, Rafaela von 2005. Weichspüler fürs Gehirn. Der Spiegel (23): 152.

Carter, Sue C. 1998. Neuroendocrine Perspectives on Social Attachment and Love. Psychoneuroendocrinology (23): 779-818.

Carter, Sue C., Jessie R. Williams, Diane M. Witt und Thomas R. Insel 1992. Oxytocin and Social Bonding. Annals of the New York Academy of Sciences (652): 204-211.

Dale, Henry H. 1906. On Some Physiological Actions of the Ergot. The Journal of Physiology (34): 163-206.

Dale, Henry H. 1909. The Action of Extracts of the Pituitary Body. Biochemical Journal (4): 427-447.

Ditzen, Beate, Marcel Schaer, Barbara Gabriel, Guy Bodenmann, Ulrike Ehlert und Markus Heinrichs 2009. Intranasal Oxytocin Increases Positive Communication and Reduces Cortisol Levels During Couple Conflict. Biological Psychiatry (65): 728-731.

De Dreu, Carsten K.W., Lindred L. Greer, Gerben A. Van Kleef, Shaul Shalvi und Michel J. J. Handgraaf 2011. Oxytocin Promotes Human Ethnocentrism. Proceedings of the National Academy of Sciences (108): 1262-1266.

Faber, Johannes 2014. Oxytocin: Die Chemie der Liebe. Badische Zeitung Online. URL: http://www.badische-zeitung.de/bildung-wissen-1/die-chemie-der-liebe-93425255. html. Zugegriffen: 25.10.2014.

Fausto-Sterling, Anne 1985. Myths of Gender: Biological Theories about Women and Men. New York: Basic Books.

Fehm, Horst L. 2001. Das Gehirn des Internisten. Zeitschrift für Wissenschaft, Forschung und Lehre an der medizinischen Universität Lübeck (18): 208-210.

Förster, Angelika 2012. Glückstankstellen: So aktivieren Sie Ihre Wohlfühlhormone. München: Heyne.

Fox Keller, Evelyn 1984. Reflections on Gender and Science. New Haven: Yale University Press.

Franzen, Martina 2011. Breaking News: Wissenschaftliche Zeitschriften im Kampf um Aufmerksamkeit. Baden-Baden: Nomos.

Goodell, Rae 1977. The Visible Scientist. Boston: Little, Brown \& Co.

Guastella, Adam J., Alexandra L. Howard, Mark R. Dadds, Philip Mitchell und Dean S. Carson 2009. A Randomized Controlled Trial of Intranasal Oxytocin as an Adjunct to Exposure Therapy for Social Anxiety Disorder. Psychoneuroendocrinology (34): 917-923.

Hagner, Michael 2008. Homo Cerebralis: Der Wandel vom Seelenorgan zum Gehirn. Frankfurt a. M.: Suhrkamp.

Haller, Lea 2012. Cortison: Geschichte eines Hormons, 1900-1955. Zürich: Chronos.

Haller, Lea, Sabine Höhler und Heiko Stoff 2014. Stress - Konjunkturen eines Konzepts. Zeithistorische Forschungen (11): 359-381.

Haraway, Donna J. 1989. Primate Visions: Gender, Race, and Nature in the World of Modern Science. New York: Routledge.

Haraway, Donna J. 1995. Die Neuerfindung der Natur: Primaten, Cyborgs und Frauen. Frankfurt a. M.: Campus.

Haraway, Donna J. 2004. The Haraway Reader. New York: Routledge.

Heinemann, Torsten 2012. Populäre Wissenschaft. Hirnforschung zwischen Labor und Talkshow. Göttingen: Wallstein.

Heinrichs, Markus, Bernadette von Dawans und Gregor Domes 2009. Oxytocin, Vasopressin and Human Social Behavior. Frontiers in Neuroendocrinology (30): 548-57.

Heuser, Uwe Jean, 2013. Hirnforscherin Singer: „Wir müssen mehr fühlen“. Die Zeit Online. URL: http://www.zeit.de/2013/23/neurowissenschaftlerin-tania-singer. Zugegriffen: 12.06.2013.

Imhof, Kurt 2006. Mediengesellschaft und Medialisierung. Medien E Kommunikationswissenschaft (54): 191-215.

Kara, Stefanie 2016. Unsere Wunderdroge. Die Zeit (15): 27-29.

Kavanagh, Anne M. und Dorothy H. Broom 1998: Embodied Risk: My Body, Myself? Social Science $\mathcal{E}$ Medicine (46): 437-444.

Kenney, Martha und Ruth Müller 2016. Of Rats and Women: Narratives of Motherhood in Environmental Epigenetics. BioSocieties (12): 23-46.

Knorr-Cetina, Karin 1984. Die Fabrikation von Erkenntnis: Zur Anthropologie der Naturwissenschaft. Frankfurt a. M.: Suhrkamp. 
Korf, Horst-Werner 2014. Gehirn und Hormone. Ernst und Berta Scharrers Konzept der Neurosekretion. Forschung Frankfurt. Das Wissenschaftsmagazin der Goethe-Universität (1): 104-107.

Kosfeld, Michael, Markus Heinrichs, Paul Zak, Urs Fischbacher und Ernst Fehr 2005. Oxytocin Increases Trust in Humans. Nature (435): 673-676.

Kretschmer, Ernst 1921. Körperbau und Charakter: Untersuchungen zum Konstitutionsproblem und zur Lehre von den Temperamenten. Berlin: Springer.

Leng, Gareth und Mike Ludwig 2015. Intranasal Oxytocin: Myths and Delusions. Biological Psychiatry (79): 243-250.

Lessenich, Stephan 2003. Soziale Subjektivität. Mittelweg (36): 80-93.

Linke, Sebastian 2007. Darwins Erben in den Medien. Eine wissenschafts- und mediensoziologische Fallstudie zur Renaissance der Soziobiologie. Bielefeld: Transcript.

Luhmann, Niklas 2007. Erkenntnis als Konstruktion. In: Niklas Luhmann (Hg.). Aufsätze und Reden. Stuttgart: Reclam: 218-242.

McCall, Cade, Nikolaus Steinbeis, Matthieu Ricard und Tania Singer 2014. Compassion Meditators Show Less Anger, Less Punishment, and More Compensation of Victims in Response to Fairness Violations. Frontiers in Behavioral Neuroscience (8): 1-10.

Merolla, Jennifer L., Guy Burnett, Kenneth V. Pyle, Sheila Ahmadi und Paul J. Zak 2013. Oxytocin and the Biological Basis for Interpersonal and Political Trust. Political Behavior (35): 753-776.

Meyer-Lindenberg, Gregor Domes, Peter Kirsch und Markus Heinrichs 2011. Oxytocin and Vasopressin in the Human Brain: Social Neuropeptides for Translational Medicine. $\mathrm{Na-}$ ture Reviews Neuroscience (12): 524-538.

Nelson, Nicole C. 2013. Modeling Mouse, Human, and Discipline: Epistemic Scaffolds in Animal Behavior Genetics. Social Studies of Science (43): 3-29.

Nickl, Roger 2006. Dossier Hormone. UZH Magazin (3): 36-39.

Nordlund, Christer 2007. Endocrinology and Expectations in 1930s America: Louis Berman's Ideas on New Creations in Human Beings. British Journal for the History of Science (40): 83-104.

Nordmann, Alfred 2005. Was ist TechnoWissenschaft? - Zum Wandel der Wissenschaftskultur am Beispiel von Nanoforschung und Bionik. Bionik: 209-218.

Oudshoorn, Nelly 1990. Endocrinologists and the Conceptualization of Sex, 1920-1940. Journal of the History of Biology (23): 163-186.

Oudshoorn, Nelly 2000. The Birth of Sex Hormones. In: Londa Schiebinger (Hg.). Feminism and the Body. Oxford: Oxford University Press: 87-117.

Paschek, Nicole 2016. Rezension: Erstaunliches Oxytocin. Spektrum.de. URL: http://www. spektrum.de/rezension/buchkritik-zu-oxytocin-das-hormon-der-naehe/1406429. Zugegriffen: 05.04.2016.

Pauly, Philipe J. 1987. Controlling Life: Jacques Loeb and the Engineering Ideal in Biology. Oxford: University Press.

Peters, Hans P., Harald Heinrichs, Arlena Jung, Monika Kallfass und Imme Petersen 2008. Medialisierung der Wissenschaft als Voraussetzung ihrer Legitimierung und politischen Relevanz. In: Renate Mayntz, Friedhelm Neidhardt, Peter Weingart und Ulrich Wengenroth (Hg.). Wissensproduktion und Wissenstransfer: Wissen im Spannungsfeld von Wissenschaft, Politik und Öffentlichkeit. Bielefeld: Transcript: 269-292.

Peters, Hans P., Joachim Allgaier, Sharon Dunwoody, Yin-Yueh Lo, Dominique Brossard und Arlena Jung 2013. Medialisierung der Neurowissenschaften: Bedeutung journalistischer Medien für die Wissenschafts-Governance. In: Edgar Grande, Dorothea Jansen, Otfried Jarren, Arie Rip, Uwe Schimank und Peter Weingart (Hg.). Neue Governance der Wissenschaft: Reorganisation - externe Anforderungen - Medialisierung. Bielefeld: Transcript: 311-335.

Planert, Ute 2007. Kulturkritik und Geschlechterverhältnis. Zur Krise der Geschlechterordnung zwischen Jahrhundertwende und „Drittem Reich“. In: Wolfgang Hardtwig (Hg.). Ordnungen in der Krise. Zur politischen Kulturgeschichte Deutschlands 1900-1933. München: Oldenbourg: 191-214.

Qintana, Daniel S. und Joshua D. Wooley 2015. Intranasal Oxytocin Mechanisms Can Be Better Understood, but its Effects on Social Cognition and Behavior Are Not to Be Sniffed At. Biological Psychiatry (79): 49-50. 
Radkau, Joachim 1998. Das Zeitalter der Nervosität: Deutschland zwischen Bismarck und Hitler. München: Carl Hanser.

Ratmoko, Christina 2010. Damit die Chemie stimmt: Die Anfänge der industriellen Herstellung von weiblichen und männlichen Sexualhormonen 1914-1938. Zürich: Chronos.

Rechter, Julia E. 1997. „The Glands of Destiny“: A History of Popular, Medical and Scientific Views of the Sex Hormones in 1920s America. PhD Thesis: Univ. of California.

Roberts, Celia 2007. Messengers of Sex: Hormones, Biomedicine and Feminism. Cambridge: University Press.

Rödder, Simone 2009. Wissenschaft auf der Titelseite der New York Times: Zur Konflikthaftigkeit medialer und wissenschaftlicher Logik. Baden-Baden: Nomos.

Rödder, Simone 2011. The Ambivalence of Visible Scientists. In: Simone Rödder, Martina Franzen und Peter Weingart (Hg.). The Sciences' Media Connection - Public Communication and its Repercussions. Dordrecht: Springer: 155-177.

Rutherford, Alexandra 2004. A „visible scientist“: B. F. Skinner's Writings for the Popular Press. European Journal Of Behavior Analysis (5): 109-120.

Sarasin, Philipp und Jakob Tanner (Hg.) 1998. Physiologie und industrielle Gesellschaft: Studien zur Verwissenschaftlichung des Körpers im 19. und 20. Jahrhundert. Frankfurt a. M.: Suhrkamp.

Savulescu, Julian und Anders Sandberg 2008. Neuroenhancement of Love and Marriage: The Chemicals Between Us. Neuroethics (1): 31-44.

Schäfer, Ingo 2015. Niemand lebt für sich allein: Warum keiner allein glücklich werden kann. Norderstedt: Books on Demand.

Schäfer, Mike S. 2007. Wissenschaft in den Medien. Die Medialisierung Naturwissenschaftlicher Themen. Wiesbaden: VS Verlag für Sozialwissenschaften.

Schaper-Rinkel, Petra 2012. Das neurowissenschaftliche Selbst. (Re)Produktion von Geschlecht in der neurowissenschaftlichen Gouvernementalität. In: Eva Sänger und Malaika Rödel (Hg.). Biopolitik und Geschlecht. Zur Regulierung des Lebendigen. Münster: Westfälisches Dampfboot: 84-106.

Scharrer, Berta 1937. Über sekretorisch tätige Nervenzellen bei wirbellosen Tieren. Naturwissenschaften (25): 131-138.

Scharrer, Ernst und Berta Scharrer 1954. Neurosekretion. In: Wolfgang Bargmann (Hg.). Handbuch der mikroskopischen Anatomie des Menschen. Bd. VI/5. Berlin: Springer: 953-1066.

Schindler, Jörg 2012a. Wir Asozialen. Der Spiegel (34): 28-29.

Schindler, Jörg 2012b. Die Rüpel-Republik: Warum sind wir so unsozial? Frankfurt a. M.: Fischer.

Schreiber, Marion 1980. Unendliches Vergnügen, unendlicher Schmerz. Der Spiegel (31): 126-139.

Sengoopta, Chandak 2006. The Most Secret Quintessence of Life: Sex, Glands, and Hormones, 1850-1950. Chicago: University of Chicago Press

Shalvi, Shaul und Karsten K.W. De Dreu 2014. Oxytocin Promotes Group-Serving Dishonesty. Proceedings of the National Academy of Sciences (111): 5503-5507.

Shen, Helen 2015. Neuroscience: The Hard Science of Oxytocin. Nature (522): 410-412.

Starling, Ernest H. 1905. Croonian Lecture: On the Chemical Correlation of the Functions of the Body I-IV. Lancet (2): 339-583.

Stein, Annett 2014. Die dunklen Seiten des Kuschelhormons Oxytocin. Die Welt Online. URL: http://www.welt.de/gesundheit/psychologie/article130360396/Die-dunklenSeiten-des-Kuschelhormons-Oxytocin.html. Zugegriffen: 20.07.2014.

Steinecke, Almut 2008. Bis die Hormone fließen. UniSpiegel (2): 32-33.

Stöcker, Christian 2005. Hormon-Trick: Nasenspray macht vertrauensselig. Spiegel Online. URL: http://www.spiegel.de/wissenschaft/mensch/hormon-trick-nasenspray-machtvertrauensselig-a-358712.html. Zugegriffen: 02.06.2015.

Stoff, Heiko 2003. Degenerierte Nervenkörper und regenerierte Hormonkörper. Historische Anthropologie (11): 224-239.

Stoff, Heiko 2004. Ewige Jugend. Konzepte der Verjüngung vom späten 19. Jahrhundert bis ins Dritte Reich. Köln: Böhlau.

Stoff, Heiko 2012. Wirkstoffe: Eine Wissenschaftsgeschichte der Hormone, Vitamine und Enzyme, 1920-1970. Stuttgart: Franz Steiner. 
Suchman, Lucy 2012. Configuration. In: Celia Lury und Nina Wakeford (Hg.). Inventive Methods: The Happening of the Social. London: Routledge: 48-60.

Tenzer, Eva 2012. Oxytocin: Das völlig überschätzte Kuschelhormon. Focus Online. URL: http://www.focus.de/wissen/bild-der-wissenschaft/tid-27680/oxytocin-dasueberschaetzte-kuschelhormon_aid_837758.html. Zugegriffen: 25.10.2015.

Venzmer, Gerhard 1933. Deine Hormone - Dein Schicksal? Von den Triebstoffen unseres Lebens. Stuttgart: Franckh'sche Verlagshandlung.

Waldby, Catherine 2000. The Visible Human Project. London: Routledge.

Weber, Jutta 2010. Making Worlds: Epistemological, Ontological and Political Dimensions of Technoscience. Poiesis and Praxis (7): 17-36.

Wehling, Peter 2016. Schüchternheit - die Entdeckung und Bekämpfung einer „Volkskrankheit". In: Roland Anhorn und Marcus Balzereit (Hg.). Handbuch Therapeutisierung und Soziale Arbeit. Wiesbaden: Springer: 495-510.

Weingart, Peter 2005. Die Wissenschaft der Öffentlichkeit: Essays zum Verhältnis von Wissenschaft, Medien und Öffentlichkeit. Weilerswist: Velbrück Wissenschaft.

Weingart, Peter 2011. Die Wissenschaft der Öffentlichkeit und die Öffentlichkeit der Wissenschaft. In: Barbara Hölscher und Justine Suchanek (Hg.). Wissenschaft und Hochschulbildung im Kontext von Wirtschaft und Medien. Wiesbaden: VS Verlag für Sozialwissenschaften: 45-61.

Wiesner, Heike 2002. Die Inszenierung der Geschlechter in den Naturwissenschaften: Wissenschafts- und Genderforschung im Dialog. Frankfurt: Campus.

Willey, Angela und Sara Giordano 2011. Why Do Voles Fall in Love? Sexual Dimorphism in Monogamy Gene Research. In: Jill A. Fischer (Hg.). Gender and the Science of Difference: Cultural Politics of Contemporary Science and Medicine. New Brunswick: Rutgers University Press: $108-125$.

Wolf, Christian 2013. Liebe ist Biochemie - Und was noch?. dasgehirn.info. URL: https:// www.dasgehirn.info/handeln/liebe-und-triebe/liebe-ist-biochemie-und-was-noch. Zugegriffen: 28.03.2016.

Wolff, Philip 2008. Psychologie: Warum wir gewalttätig werden. Spiegel Online. URL: http:// www.spiegel.de/wissenschaft/mensch/psychologie-warum-wir-gewalttaetig-werdena-579665.html. Zugegriffen: 03.10.2016.

Sabine Maasen

TUM School of Governance, Friedrich Schiedel-Stiftungslehrstuhl für Wissenschaftssoziologie

Technische Universität München

Arcisstr. 21

80333 München

Deutschland

sabine.maasen@tum.de 